\title{
Nouvelle espèce d'Opbidascaris Baylis 1921, parasite du serpent crotale (Crotalus durissus terrificus)
}

\author{
par Paulo ARAUJO
}

(Laboratoire de Parasitologie, Faculté de Pharmacie et Biochimie, Université de São Paulo, São Paulo, Brésil)

\section{Résumé}

Description d'une nouvelle espèce du genre Ophidascaris, O. sprenti n. sp., parasite du serpent crotale (Crotalus durissus terrificus) du Brésil. Les caractères présentés permettent de la distinguer d'un grand nombre d'espèces de l'Ancien et du Nouveau Monde.

\section{Summary}

Description of a new species of the genus Ophidascaris, $O$. sprenti n. sp., parasite of the rattle snake (Crotalus durissus terrificus) of Brazil. The characters described allow to distinguish this species from a large number of species already described from the New and Old World.

Dans l'estomac d'un serpent Crotalus durissus terrificus, mort à l'Institut Butantan, São Paulo (nécroscopie $\mathrm{n}^{\circ} 1610$ ), nous avons trouvé 36 exemplaires de Nématodes adultes ayant les caractères du genre Ophidascaris, mais morphologiquement différents des autres espèces décrites.

Comme probablement il s'agit d'une espèce nouvelle, nous proposons le nom d'Ophidascaris sprenti $\mathrm{n}$. sp. 


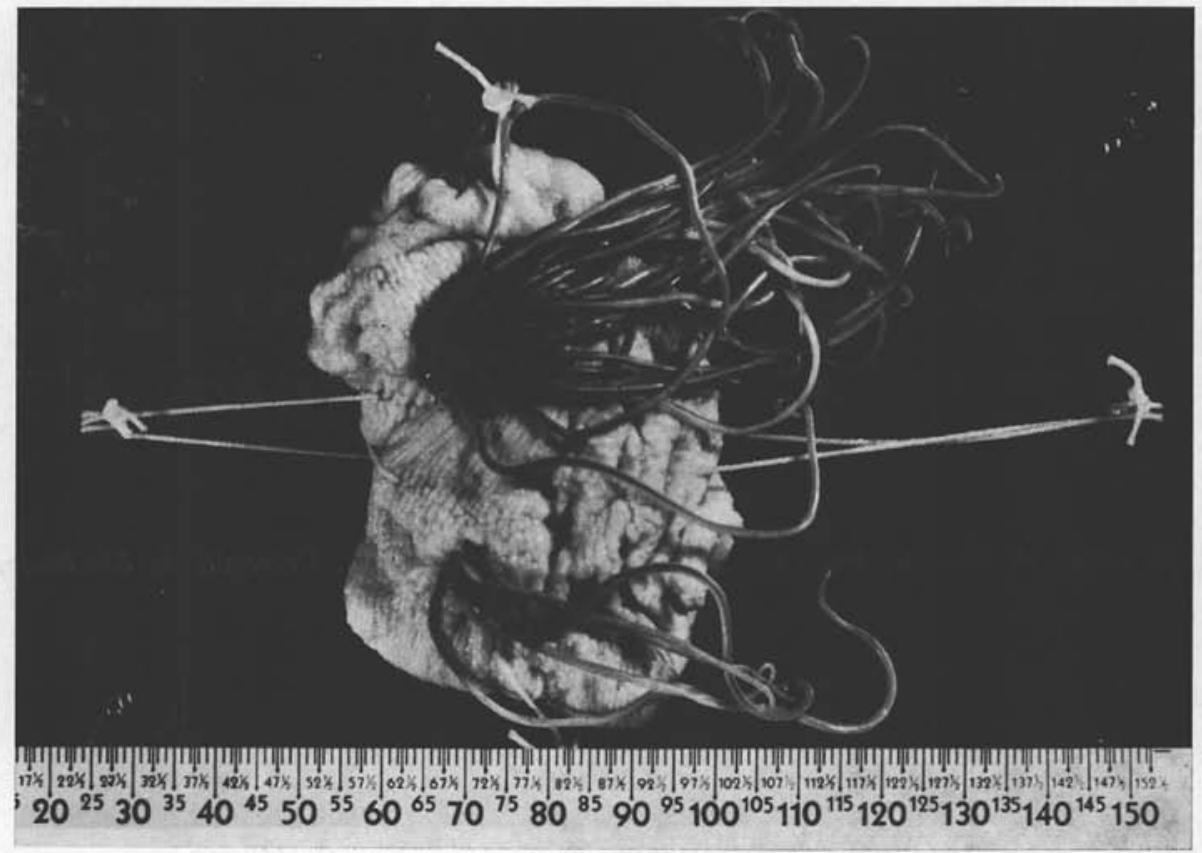

Fig. 1. - Fragment d'estomac de Crotalus durissus terrificus contenant dans deux orifices l'extrémité antérieure de plusieurs exemplaires d'Ophidascaris sprenti $\mathrm{n}$. sp.

\section{OPHIDASCARIS SPRENTI n. sp.}

\section{HôTE : Crotalus durissus terrificus.}

Localisation: Estomac. Les vers étaient distribués en deux groupes dans deux orifices de la paroi gastrique, plus nombreux dans l'un que dans l'autre (fig. 1). Ils se présentaient avec l'extrémité antérieure profondément enfoncée dans la paroi gastrique. Cette particularité des vers de se présenter fixés à la surface interne de l'estomac, selon la citation de Sprent et Mines (1960), est aussi rencontrée fréquemment chez $O$. filaria (Dujardin, 1845) et chez Polydelphis anoura Dujardin, 1845, mais cette dernière espèce se localise dans l'intestin de serpent. L'espèce Amplicaecum robertsi Sprent et Mines, 1960, présente aussi ce mode de fixation dans l'estomac de serpent; la photographie de cette espèce donnée par Sprent et Mines (1960) peut être superposée à notre figure $\mathrm{n}^{\circ} 1$ relative à $O$. sprenti $\mathrm{n}$. $\mathrm{sp}$.

Distribution géOgRAPHIQUE: Brésil.

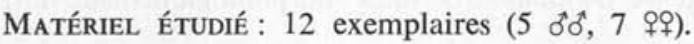

DESCRIPTION: Longueur: mâles 59-66 mm; femelles 81-101 mm. Largeur . mâles $0,7-0,9 \mathrm{~mm}$; femelles $1,2-1,4 \mathrm{~mm}$. 
Fig. 2. - Extrémité antérieure d'une femelle d'O. sprentı n. sp. montrant l'interlèvre ventrale.

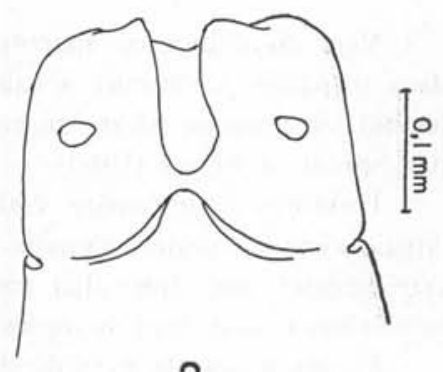

2

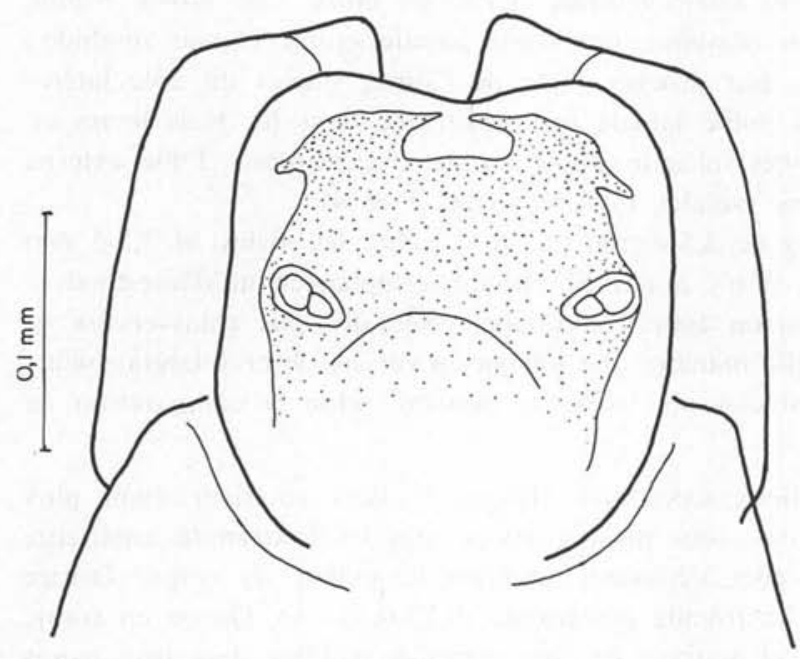

FIG. 3. - Lèvre dorsale

d'O. sprenti n. sp. femelle.

3

FIG. 4. - Lèvre latéro-ventrale d'O. sprenti n. sp. femelle montrant les deux papilles (l'une grande et l'autre petite) et l'amphide en haut et à droite.

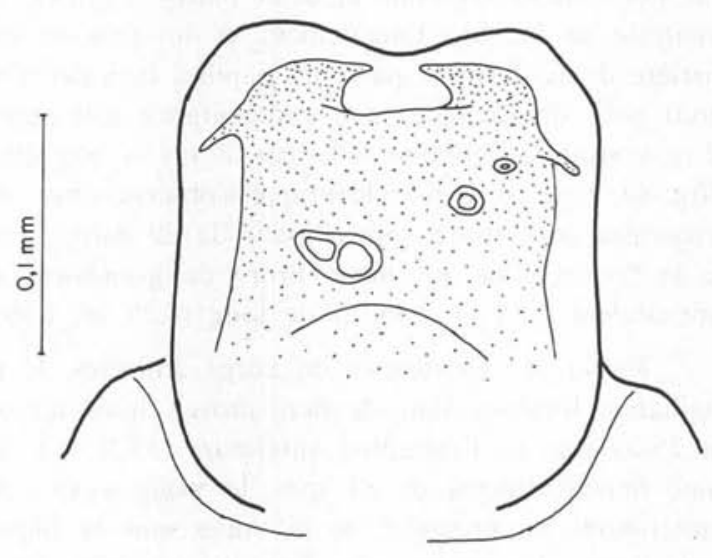

4

Annales de Parasitologie humaine et comparée (Paris), t. 44, 1969, $\mathrm{n}^{\circ} 4$ 
Vers de coloration marron dans la partie antérieure, rougeâtre et plus claire dans ia partie postérieure à cause de la présence des organes reproducteurs. Latéraiement, on aperçoit un dessin en arête de poisson, également observé chez $A$. robertsi par Sprent et Mines (1960).

Lextrémité antérieure comporte trois lèvres rectangulaires, plus longues que larges, avec les angles arrondis et armées à leurs bords libres d'une denticulation extrêmement fine. Interlabia petits, à bords antérieurs concaves (fig. 2). Les sillons sous-labiaux sont bien marqués.

La lèvre dorsale possède deux grosses papilles, chacune avec deux terminaisons nerveuses (fig. 3). Chaque lèvre latéro-ventrale porte, en outre, une grosse papille également à double terminaison nerveuse, une petite papille simple et une amphide ; ces deux dernières formations sont proches l'une de l'autre, situées du côté latérodorsal de la lèvre (fig. 4). La pulpe labiale est symétrique dans les trois lèvres et envoie antérieurement deux lobes, chacun divisé en deux expansions, l'une externe et l'autre interne; ramifications radiales invisibles (fig. 3 et 4).

Esophage claviforme, long de 2,5-4 mm (4,2-6 \%) chez les mâles, et 3,5-5 mm $(4,3-5,1 \%)$ chez les femelles. Il n'y a pas de caecum œsophagien ni d'intestinal.

intestin de coloration marron foncé, rectiligne, avec des plis transversaux et obliques (fig. 5), disposés de telle manière que lorsque le ver est observé latéralement, ils montrent un aspect semblable à une arête de poisson, selon la comparaison de Sprent et Mines (1960).

Mâle: Corps avec les deux extrémités effilées, le tiers antérieur étant plus mince. Les branches testiculaires vont jusqu'à 10-29 $\mathrm{mm}$ de l'extrémité antérieure $(16,3-43,7 \%)$, c'est-à-dire qu'elles dépassent toujours le milieu du corps. Orifice cloacal à $0,144-0,192 \mathrm{~mm}$ de l'extrémité postérieure $(0,23-0,31 \%)$. Queue en forme de cône, sans mucron terminal et pourvue de cinq paires de papilles, dont trois paires en situation sub-ventrale et deux paires latérales. La première paire de papilles subventrale se localise latéralement et un peu en arrière de l'ouverture cloacale. En arrière de la dernière paire de papilles latérales s'ouvrent les phasmides. En avant et tout près du cloaque, une protubérance qui peut simuler une papille ne doit pas être considérée comme telle car il n'y a pas été observé de terminaison nerveuse (fig. 6). Une formation identique s'observe chez Amplicaecum robertsi. Papilles précloacales en nombre approximatif de 39 paires. Les spicules, sans particularité quant à la forme, sont du même ordre de grandeur; sur un mâle long de $66 \mathrm{~mm}$, ils mesuraient 2,82 et $2,88 \mathrm{~m}$ de long $(4,27$ et $4,36 \%)$.

Femelle: Extrémités du corps amincies, le tiers antérieur plus fin. Vulve peu saillante, localisée dans le tiers moyen mais toujours en avant du milieu du corps, à 25-39 $\mathrm{mm}$ de l'extrémité antérieure (33,3-39,3\%). Vagin dirigé vers l'arrière ; sur une femelle longue de $81 \mathrm{~mm}$, le vagin avait $1,608 \mathrm{~mm}$. Les branches ovariennes antérieures s'approchent de la vulve sans la dépasser; en arrière, elles n'atteignent pas l'anus. La queue, plus longue que celle des mâles, est conique et n'a pas de mucron terminal (fig. 7). Sa longueur est de 0,480-0,756 mm (0,5-0,8\%). Les œufs 
sont sub-sphériques; ils mesurent $0,068-0,093 \times 0,058-0,074 \mathrm{~mm}$ et présentent une fine granulation externe.

\section{Diseussion.}

Le genre Ophidascaris contient jusqu'à présent 24 espèces décrites, trouvées chez les ophidiens, les lacertiliens $(O$. filaria) et chez les oiseaux ( $O$. crassilabiata Schuurmans Stekhoven, 1950). Chez les amphibiens ont été rencontrées par Walton (1937) seulement des larves enkystées d'O. labiatopapillosa Walton (1927).

Il y a 8 espèces américaines :

1) O. arndti Sprehn, 1929, in Lachesis lanceolatus; Amérique du Sud et in Xenodon merremii ; Brésil.

2) O. crassilabiata Schuurmans Stekhoven, 1950, in Notiopsar cinereus; Détroit de Magellan.

3) O. labiatopapillosa Walton, 1927, in Coluber constrictor constrictor; Floride (U.S.A.).

4) O. obconica (Baird, 1860), in Helicops angulatus; Brésil.

5) O. ochoterenai Caballero, 1939, in Drymarchon corais melanurus; Mexique.

6) O. sicki Freitas, 1951, in Xenodon merremii, X. severus; Brésil.

7) O. travassosi Vaz, 1938, in Crotalus terrificus; Brésil.

8) O. trichuriformis Vaz, 1935, in Liophis miliaris, Crotalus terrificus, Xenodon merremii ; Brésil. Aussi in Erythrolamprus brizonus ; Panamá et in Lystrophis dorbigny ; Uruguay.

Les espèces sud-américaines sont: $O$. arndti, $O$. crassilabiata, $O$. obconica, $O$. sicki, $O$. travassosi et $O$. trichuriformis.

Les 16 autres espèces, citées dans l'Ancien Monde, sont :

9) O. ajgaris Khera, 1956, in Python molurus; Indes.

10) O. amucronata Schuurmans Stekhoven, 1937, in Python sebae, Bitis arietans; Afrique.

11) O. baylisi Robinson, 1934, in Python reticulatus; Malaisie.

12) O. filaria (Dujardin, 1845), in Python, Astrophis, Bitis, Boaedon, Naja, Aspidites, Dasypeltis, Varanus; Indes, Afrique, Malaisie.

13) O. genoheteromegala Kreis, 1938, in Coluber quatuorlineatus; Iles Salomon.

14) O. gestri (Parona, 1890), in Tropidonotus quincunciatus; Indes, Burma.

15) O. intorta (Gedoelst, 1916), in Bitis spp., Naja nivea; Afrique.

16) O. microspicula Kreis, 1938, in Naja tripudians; Asie Tropicale.

17) O. mombasica Baylis, 1938, in Psammophis, Ablabophis, Crotaphopeltis; Afrique.

18) O. najae (Gedoelst, 1916), in Naja et Bungarus spp.; Afrique, Indes.

19) O. natricis Yamaguti, 1935, in Natrix tigrina et Elaphe quadrivirgata; Japon. Aussi in serpent aquatique; Russie. 


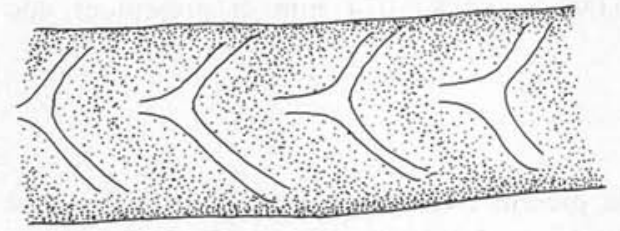

FIG. 5. - Intestin d'O. sprenti n. sp. montrant les p'is transversaux et obliques

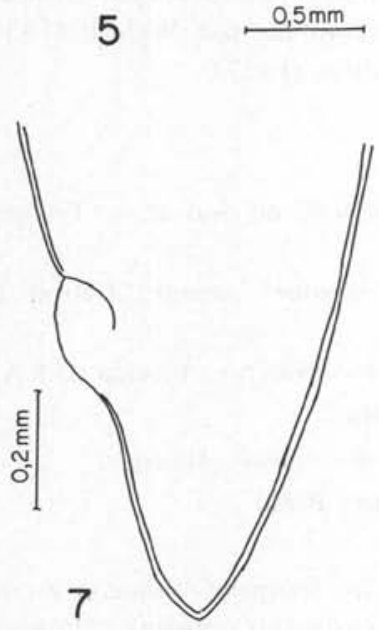

FIG. 7. - Queue de femelle d'O. sprenti n. sp.

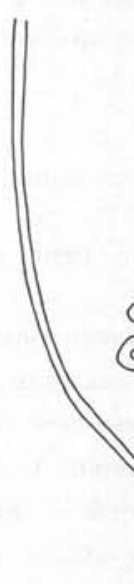

Fig. 6. - A) Vue latérale d'une queue de mâle d'O. sprenti n. sp. B) Détail de la protubérance pré-cloacale sans terminaison nerveuse

20) O. papillifera (Linstow, 1898), in serpent ; Archipel de Bismark.

21) O. pyrrhus Johnston et Mawson, 1942, in Pseudechis porphyriacus, Demansia psammophis, Notechis scutatus; Australie.

22) O. radiosa (Schneider, 1866), in Bitis gabonica, Causus rhombeatus; Afrique.

23) O. solitaria (Linstow, 1903), in Dipsadomorphus dendrophilus; Siam.

24) O. solenopoion Chabaud, 1960, in Ithycyphus miniatus; Madagascar. suivants :

$O$. sprenti n. sp. se différencie des autres espèces du genre par les caractères

Chez $O$. filaria, $O$. amucronata, $O$. baylisi, $O$. genoheteromegala, $O$. labiatopapililosa, O. mombasica, O. natricis, O. obconica, O. ochoterenai, O. pyrrhus, $O$. radiosa, $O$. solenopoion, $O$. sicki et $O$. trichuriformis la vulve se situe en arrière du milieu du corps tandis que chez $O$. sprenti n. sp., elle se localise en avant du milieu du corps.

Chez $O$. crassilabiata, à cause de la description incomplète présentée par Schuurmans Stekhoven (1950), la localisation de la vulve est inconnue ; cependant, l'auteur 
affirme que la queue du mâle et celle de la femelle sont identiques et que l'hôte est un oiseau (Notiopsar cinereus). Chez O. sprenti n. sp., les queues du mâle et de la femelle sont sensiblement différentes et l'hôte est un serpent.

O. gestri, selon Baylis (1921), présente la vulve localisée dans le tiers antérieur, mais la queue du mâle et même celle de la femelle sont pourvues d'un mucron termınal; la queue est très courte chez la femelle. Chez O. sprenti n. sp., dans les deux sexes, la queue n'a pas de mucron terminal et, chez la femelle, elle est relativement longue $(0,5-0,8 \%$ de la longueur du ver).

Chez $O$. intorta, d'après Schuurmans Stekhoven (1937), la pulpe labiale est ramifiée antérieurement; la queue de la femelle est courte, ronde, sans mucron termınal et plus petite que celle du mâle; les spicules sont fortement inégaux (2 et $0,8 \mathrm{~mm}$ ). $O$. sprenti n. sp. présente une pulpe labiale non ramifiée et la queue de la femelle, dépourvue de mucron terminal, est plus longue que celle du mâle; les spicules sont sub-égaux.

Chez O. microspicu'a, la vulve se localise en avant du second tiers du corps [Kreis (1938) signale : vulve $=55,8-62,8 \%$ ] ; la queue du mâle comme celle de la femelle est courte et ronde ; celle du mâle possède un mucron terminal qui mesure $0,034 \mathrm{~mm}$ de longueur. Chez $O$. sprenti n. sp., la vulve est située dans le second tiers du corps; la queue du mâle comme celle de la femelle est conique; la queue du mâle est dépourvue de mucron terminal.

Chez O. najae, selon Baylis (1921), la vulve se trouve au milieu du corps, les spicules représentent 8,1 et $7,4 \%$ de la longueur du corps et la queue du mâle possède 6 paires de papilles post-cloacales ( 4 sub-ventrales et 2 latérales). Chez $O$. sprenti n. sp., la vulve est localisée en avant du milieu du corps, les spicules représentent 4,27 et $4,36 \%$ de la longueur du corps et chez les mâles il y a 5 paires de papilles post-cloacales ( 3 paires sub-ventrales et 2 paires latérales).

$O$. papillifera, selon Baylis (1921), présente une queue courte et arrondie chez le mâle comme chez la femelle; la queue des mâles a 6 paires de papilles post-cloacales, dont une paire de papilles petites localisée tout près et en arrière de l'orifice cloacal, et située aux environs de la ligne médiane. Chez $O$. sprenti n. sp., la queue du mâle comme celle de la femelle est conique ; celle du mâle n'a que 5 paires de papilles, la paire moyenne post-cloacale manquant.

Chez O. solitaria, d'après la description sommaire par Linstow (1903) d'une femelle immature, citée par Baylis (1921), la queue et l'œsophage représentent 0,38 et $6,6 \%$ de la longueur du corps, respectivement, tandis que chez $O$. sprenti n. sp., ces pourcentages sont de $0,5-0,8 \%$ et $4,3-5,1 \%$, respectivement.

$O$. travassosi présente la vulve au milieu du corps, 7 paires de papilles postcloacales et la queue de la feme'le, courte, représente $0,3 \%$ de la longueur du corps. Chez $O$. sprenti n. sp., la vulve se situe en avant du milieu du corps, les mâles possèdent 5 paires de papilles post-cloacales et la queue de la femelle représente 0,5 à $0,8 \%$ de la longueur du corps.

Nous n'avons pas pu obtenir des renseignements sur la morphologie d'O. ajgaris. 
Selon citation de Yamaguti (1961), cet ascaride a été trouvé dans la bouche et la cavité générale de Python molurus aux Indes. Cependant, nous croyons qu'il s'agit d'une espèce différente d'O. sprenti n. sp., en raison de la distribution géographique et des hôtes qui sont différents.

Quant à l'O. arndti, nous l'avons laissé en dernier parce que cette espèce est celle qui, morphologiquement, se rapproche le plus d'O. sprenti n. sp. et aussi parce qu'e'le a été trouvée chez des serpents du Brésil. Cependant, ces deux espèces peuvent être différenciées par les caractères suivants:

Chez $O$. arndti, d'après la description originale de Sprehn (1929), la vulve se situe au milieu du corps, en général un peu en arrière, quelquefois un peu en avant ; dans ce cas extrême, sa situation se trouve à $45,8 \%$ de la longueur du corps. Chez les mâles, il y a une grande papille près du bord antérieur du cloaque. Selon la redescription de l'espèce faite par Freitas (1955), la vulve se situe dans le tiers moyen, en avant ou en arrière du milieu du corps. Chez les mâles, le testicule se plie en forme de « $U$ » en avant ou en arrière du milieu du corps, à une distance variable de 27,77 à $30,22 \mathrm{~mm}$ de l'extrémité antérieure et se termine plus bas. Cependant, les longueurs extrêmes des mâles fournies par Freitas (1955) sont 46,03 et $51,82 \mathrm{~mm}$. Ainsi, on vérifie que le testicule n'atteint jamais le milieu du corps, étant placé toujours dans la moitié postérieure. En outre, d'après Freitas (1955), et confırmant Sprehn (1929), chez les mâles, il y a une papille moyenne située sur le bord antérieur de l'ouverture cloacale et, de plus, selon Freitas (1955), la queue des mâles se termine par une petite pointe conique qui mesure 0,039 à $0,043 \mathrm{~mm}$ de longueur. Chez $O$. sprenti n. sp., la vulve est toujours localisée en avant du milieu du corps $(33,3-39,3 \%)$; chez le mâle, il n'y a pas de papille impaire précloacale; les branches testiculaires dépassent le milieu du corps, atteignant quelquefois le tiers antérieur $(16,3-43,7 \%)$; la queue des mâles ne possède pas la pointe conique mentionnée par Freitas (1955).

\section{Bibliographie}

AsH (L. R.) et BEAVER (P. C.), 1963. - Redescription of Ophidascaris labiatopapillosa Walton, 1927, an ascarid parasite of North American snakes. J. Parasit., Lancaster, 49 : 765-770.

Baylis (H. A.), 1921. - On the classification of the Ascaridae. II. The Polydelphis group; with some account of the other ascarids parasitic in snakes. Parasitology, Cambridge, 12 : 411-426.

Caballero y (C. E.), 1939. - Nemátodos de los reptiles de México. III. An. Inst. Biol., Univ. Nac. México, México, 10:73-82.

Chabaud (A.-G.), 1960. - Deux nématodes parasites de serpents Malgaches. Mém. Inst. Scient. Madagascar, Tananarive, 14: 95-103.

Freitas (J. F. T.), 1951. - Ophidascaris sicki n. sp. (Nematoda, Ascaroidea). Rev. Brasil. Biol., Rio-de-Janeiro, 11 : 255-258. 
—, 1955. - Redescrição de Ophidascaris arndti Sprehn, 1929 (Nematoda, Ascaroidea). Rev. Ibérica Parasit., Granada, Tomo Extra: 17-22.

Johnston (T. H.) et MAwson (P. M.), 1942. - The Gallard collection of parasitic nematodes in the Australian Museum. Rec. Aust. Mus., Sydney, $21:$ 110-115.

KreIS (H. A.), 1938. - Beiträge zur Kenntnis parasitischer Nematoden. VIII. Neue parasitische Nematoden aus dem Naturhistorischen Museum Basel. Ctbl. Bakt. Orig., 142 : 329-352.

Robinson (V. C.), 1934. - On a collection of parasitic worms from Malay. I. Nematodes (superfamilies Ascaroidea and Oxyuroidea). Parasitology, Cambridge, 25: 481-488.

Schuurmans Stekhoven Jr. (J. H.), 1937. - Parasitic nematoda. Inst. Parcs. Nat. Congo Belge, Bruxelles, $4: 1-40$.

—, 1950. - Nematodos parasitarios del Chaco Paraguayo y de Argentina del Museo de Estocolmo. Acta Zool. lilloana, Tucumán, 9: 325-345.

SPrehn (C. E. W.), 1929. - Eine neue Ascaride, Ophidascaris arndti n. sp. aus einer südamerikanischen Schlange. Zool. Anz., 83 : 280-282.

SPRENT (J. F. A.) et Mines (J. J.), 1960. - A new species of Amplicaecum (Nematoda) from the carpet snake (Morelia argus variegatus): with a re-definition and a key for the genus. Parasitology, Cambridge, 50 : 183-198.

VAZ (Z.), 1935. - Lesões produzidas no estômago de cobras por novo nematoide Ophidascaris trichuriformis n. sp. Ach. Inst. Biol., São Paulo, 6: 41-44.

—, 1938. - Nova espécie do gênero Ophidascaris parasita da cascavel (Crotalus terrificus). In Livro Jubilar do Professor Lauro Travassos, Rio-de-Janeiro, p. 495-500.

Walton (A. C.), 1937. - The nematoda as parasites of Amphibia. III. Studies on life histories. J. Parasit., Lancaster, $23:$ 299-300.

YAMAGUTI (S.), 1935. - Studies on the helminth fauna of Japan. XI. Reptilian nematodes. Jap. J. Zool., Tokyo, 6: 393-402.

-, 1961. - The nematodes of vertebrates. In Yamaguti (S.), Systema helminthum. New York, Interscience Publishers, Inc., v. 3, pt. 1 et 2, p. 162-163. 